\title{
An investigation of collisions of liquid coated particles
}

\author{
Megan Danczyk ${ }^{1}$, Luke Fullard ${ }^{2}$, and Daniel Holland ${ }^{1 *}$ \\ ${ }^{1}$ Department of Chemical and Process Engineering, University of Canterbury, New Zealand \\ ${ }^{2}$ School of Fundamental Sciences, Massey University, New Zealand
}

\begin{abstract}
The presence of even small amounts of liquid between particles dramatically changes the dynamics of collisions. This study considers granular collisions between two particles coated with a thin layer of viscous liquid, such that the capillary number is high and viscous forces dominate. High-speed particle tracking velocimetry was used to experimentally study the collisions of two smooth spheres with and without liquid coatings. We then use these experiments to examine four theoretical models that describe the collisions. A key challenge when modelling viscous forces is that the force which is predicted as particles approach each other scales with the inverse of the distance, i.e. tends to infinity. Most existing models impose a limit to the viscous force. One recent model instead assumes a hard sphere collision. These fundamentally different approaches produce different rebound outcomes. A fair match between experiments and simulations was obtained when using the hard sphere collision model, but only if an empirically-fitted glass transition pressure model was used to describe the minimum approach distance.
\end{abstract}

\section{Introduction}

Here we consider Discrete Element Method (DEM) simulations of granular flow in the pendular regime, where there are cohesive forces acting through the liquid bridges formed at contact points between granules [1]. Liquid is present in many granular flows, including both natural [2] and industrial systems [3]. DEM simulations are able to model dry granular flow quite accurately [4], but are limited when describing systems containing small amounts of liquid [5]. A variety of contact models exist that describe wet particle collisions in the pendular regime, which include a capillary and viscous cohesive force for the liquid [6-8]. The capillary force models are quite well established [9]. The viscous force models differ in terms of the functional form, and/or the approach used to describe the forces when particles almost touch. In this study, we present detailed measurements of collisions between two particles coated in a thin layer of viscous liquid using a system similar to the so-called Stokes' cradle set up $[10,11]$. These measurements provide the basis for critically examining four models of wet particle collisions and will help to establish DEM as a robust technique for studying wet granular flows.

For unsaturated wet granular flows, the primary additional contact forces considered are the capillary and viscous forces. The capillary number is used to find the relative influence of viscous and capillary forces, and is defined as:

$$
C a=\frac{3 \mu v_{r} r_{e f f}}{\sigma h_{o}}
$$

where $\mu$ is the dynamic viscosity of the liquid, $v_{r}=$ $v_{1}-v_{2}$ is the relative velocity between two colliding particles, $r_{e f f}$ is the effective radius defined by $r_{e f f}=$

\footnotetext{
* Corresponding author: daniel.holland@ canterbury.ac.nz
} A video is available at https://doi.org/10.48448/wyee-sq13 
fourth rebound mechanism has been proposed in which the liquid goes through a glass transition arising from the increase in the liquid pressure as the particles approach [6]. It is the glass-like behaviour of the liquid that causes a rebound.

The aforementioned theoretical models for wet particle collisions in the pendular regime have been individually implemented in DEM $[8,14,18]$. Experimental validation of these models has been limited to observations of the macroscopic qualities such as avalanche amplitude and discharge mass flow $[19,20]$. Although qualitative predictions of the model are good, where discrepancies in the bulk dynamics remain, it is difficult to identify the cause of these differences in such complex systems. To the best of our knowledge, no direct comparison of these models with experimental measurements of individual collisions has been presented, and hence it is unclear which approach is best suited for use in simulations.

In this study, we examine four wet granular flow models in the pendular regime. First, we outline the viscous contact models used in this study. Then, the experimental methods used to track the collisions experimentally are described. Finally, we compare the DEM models with the experimental measurements.

\section{Theory}

The governing equations for the discrete element method are derived from Newton's laws of motion. Here, the open-source DEM software, LIGGGHTS ${ }^{\mathrm{TM}}$ was used [21].

The forces considered in the pendular regime due to the cohesion effect of the liquid surrounding the particle are the capillary force $F_{c, i j}^{n}$ and $F_{c, i j}^{t}$, and the viscous force $F_{v, i j}^{n}$ and $F_{v, i j}^{t}$. Generally, these forces are considered in both the normal $n$, and tangential $t$ directions. In the present work the collisions are linear so the tangential forces are zero [22]. Also, the capillary forces are negligible for the thin films and high viscosity oil considered here. Fig. 1 illustrates the key variables used to define the collision, including $\delta_{i}$ the oil thickness on particle $i$ and $h_{i j}$ the separation distance between particles, which is defined as the point in the middle of the liquid bridge at the nearest point of solid surface contact between particle $i$ and $j$.

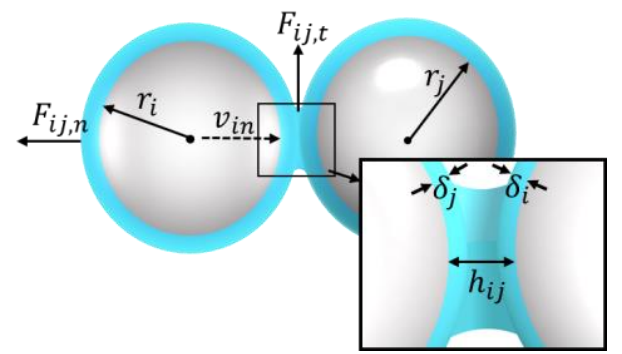

Fig. 1. Schematic of two spheres colliding.

There are two models implemented in the core LIGGGHTS source code [8, 14]. Both of these models use a solid-solid contact model for the rebound mechanism. In one model, referred to as the Easo model, no contact forces are considered until the solid-solid contact has been initiated. For the second model, referred to as the Washino model, the viscous forces are considered as soon as the liquid films on the surface of the particles touch. Both models use the viscous model proposed by Nase [7]:

$$
F_{v, i j}^{n}=6 \pi \mu r_{e f f} v_{r} \frac{r_{e f f}}{h_{i j}} .
$$

Here the effective radius is $r_{e f f}=\frac{r_{i} r_{j}}{\left(r_{i}+r_{j}\right)}$, the relative velocity is $v_{r}=v_{i}-v_{j}$, the viscosity of the oil is $\mu$, and $h_{i j}$ is the distance between the solid surfaces of the particles at the current timestep. In both of these models, the viscous force is limited by assuming a minimum value for $h_{i j} \geq h_{\min }$ when calculating the viscous force. Here we choose the recommended values for $h_{\min }$ of $1.01 r_{E f f}$ and $1.01 r_{E f f}$ for the Washino and Easo models, respectively.

The third model considered is referred to as the Davis OG model [16]. It uses a different form of the viscous force to account for the curvature of the surface. Further, when the particles approach to within a minimum separation distance, they undergo a hard sphere collision to enforce rebound:

$$
F_{v, i j}^{n}=\left\{\begin{array}{cc}
6 \pi \mu r_{e f f} v_{r} \frac{\left(1-\frac{h_{i j}}{h_{\max }}\right)^{2}}{h_{i j}} & h_{\text {min }}<h_{i j} \\
-e m_{\text {eff }} \frac{v_{r}}{\Delta t} & h_{\text {min }} \geq h_{i j}
\end{array}\right.
$$

where $e$ is the coefficient of restitution, $\Delta t$ is the timestep of the simulation $\left(1 \times 10^{-7} \mathrm{~s}\right)$, and $h_{\max }=$ $\left(r_{i}\left(L C_{i}\right)^{\frac{1}{3}}-1\right)+\left(r_{j}\left(L C_{j}\right)^{\frac{1}{3}}-1\right)$ in which $L C_{i}$ is the initial surface liquid content on the $i$ th particle. The experimentally measured oil thickness is used to calculate $L C_{i}$. For the Davis OG model, when the particles approach to within a distance $h_{\text {min }}$, the particles undergo a hard sphere rebound. We use $h_{\text {min }}=$ $0.2 h_{o}$ as a best fit rebound criteria for both viscosities.

Davis et al. [22] noted that if a fixed value was used for $h_{\text {min }}$, the match to experiments was poor. They proposed a modification of their model, here referred to as the Davis PGT model, where the minimum separation distance is based on the glass transition pressure,

$$
h_{\text {min }}=h_{g}=\left(\frac{3 \mu r_{e f f} v_{r}}{P_{G T}}\right)^{1 / 2}
$$

where $P_{G T}$ is the glass transition pressure of the silicone oil. The glass transition pressure for high viscosity lubricants is typically around $300 \mathrm{MPa}$ [23]. However, we use a much smaller value (5-7 MPa) to fit the data. One reason for this could be due to the increasing viscosity of the oil as the solid spheres approach.

\section{Methods}

The experimental setup is based on the so-called Stokes' cradle experiment [11] and is shown in Fig. 2. The string is a $20 \mathrm{lb}$ fishing line. The striker ball rests on a jack with a hinged plate activated by a spring action to 
release the sphere. We refer to the striker sphere as sphere 1 and the initially stationary sphere as sphere 2 . Two different types of steel spheres were used in these tests, stainless steel grade 316 (SS), and chrome steel grade 100 (CS). The SS was measured to have a density of $7960 \pm 1 \mathrm{~kg} \mathrm{~m}^{-3}$ and the CS had a density of $7794 \pm 2$ $\mathrm{kg} \mathrm{m}^{-3}$. All of the spheres had a diameter of $25.4 \mathrm{~mm}$. The dry coefficients of restitution were measured to be $0.9 \pm 0.1$ for the SS and $0.95 \pm 0.05$ for the CS. Sphere 2 was completely coated in oil by immersing it in an oil bath. After 2 minutes of dripping for the $3000 \mathrm{cP}$ oil and 3 minutes of dripping for the $10000 \mathrm{cP}$ oil, the camera began recording and the striker particle was released. The oil thickness was found to be $0.20 \pm 0.03 \mathrm{~mm}$ for the $3000 \mathrm{cP}$ oil and $0.28 \pm 0.03 \mathrm{~mm}$ for the $10000 \mathrm{cP}$ oil.

The collisions were captured using a Photron SA5 high speed camera and a Tamron SP AF Aspherical XR lens. The pictures were taken at 5000 frames per second with focal length of $75 \mathrm{~mm}$, a fully open exposure, and aperture of $F / 4$. The displacement data of the spheres was found using the particle tracking velocimetry (PTV) program, GOM correlate $\AA$. The diameter of the dry striker sphere was used to calibrate the pixel size in each set of images, which gave a resolution of about $0.1 \mathrm{~mm}$ per pixel.

The simulations were set up to replicate the experimental Stokes' cradle system. The simulations use the measured densities, diameter, and coefficients of restitutions. Additionally, the Young's Modulus was specified as $2 \times 10^{11} \mathrm{~Pa}$ for both materials, and the Poisson's Ratio was set to 0.28 and 0.35 for the CS and SS respectively. A time step of $1 \times 10^{-7} \mathrm{~s}$ was used and collisions were simulated for a total time of $10 \mathrm{~ms}$.

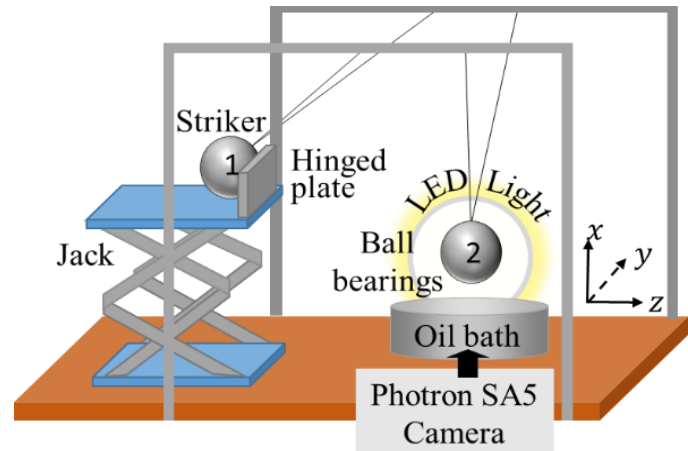

Fig. 2. Experimental Stokes' cradle setup

\section{Results}

Fig. 3 shows the displacement in $z$ for two collisions for the experimental and simulated data. For all the datasets, the initial position was defined as the point when the oil films are first touching. Data are shown for both the striker sphere (Sphere 1) and the initially stagnant sphere (Sphere 2). The experimental data are displayed using the symbols. The simulation results are shown using lines, with solid lines representing the displacement of the striker and the dashed lines representing the displacement of the initially stagnant oil coated sphere.

The Stokes' number is used to compare the data. The Stokes' number is a ratio of the inertial to viscous forces

$$
S t=\frac{m v_{r, i n}}{\left(6 \pi \mu r_{e f f}^{2}\right)}
$$

where $v_{\mathrm{r}, \text { in }}$ is the initial relative velocity. The collisions considered in Fig 3 are for Stokes' numbers of 2.7 and 3.1 , which corresponds to an initial velocity of $0.18 \mathrm{~m} \mathrm{~s}$ ${ }^{1}$ and $0.22 \mathrm{~m} \mathrm{~s}^{-1}$, respectively. These experiments show two outcomes - agglomeration and rebound. The collision is deemed to occur at the point where the acceleration reached a maximum, approximately $1 \mathrm{~ms}$ after the collision is initiated. After the collision ends at about $2 \mathrm{~ms}$, the two spheres move at a steady post collision velocity.

In the experimental data in Fig. 3a, at $\mathrm{St}=2.7$, the collision resulted in an agglomeration of the spheres. Due to the frame rate, the initial contact of the liquid film could be off by up to a $0.2 \mathrm{~ms}$. However, it is clear that the rebound occurs at about $1 \mathrm{~ms}$. After the collision, the velocities of the spheres are the same denoting an agglomeration. Fig. $3 \mathrm{~b}$ shows a collision at $\mathrm{St}=3.1$. The collision is completed faster than the collision shown in Fig 3a. The rebound occurs at about $0.75 \mathrm{~ms}$ and resulted in a separation of the two spheres, which can be seen by the different velocities of the particles after the collision.

When compared with the simulated data, all four sets of simulations produce a similar response. However, only the Davis PGT model, that uses the glass transition pressure rebound, predicted agglomeration for $\mathrm{St}=2.7$ (see Fig. 3a). The rebound in the simulations using the Davis models also occurs sooner than in the Easo and Washino models, similar to the collision mechanics seen experimentally. The Washino or Easo models don't rebound until after the solid surfaces have contacted, so the rebound is slightly delayed compared with the Davis OG and Davis PGT models. The post collisional velocities of the Easo, Washino, and Davis OG model are reasonable but are not able to predict the Stokes' number at which the collision transitions from agglomeration to separation.

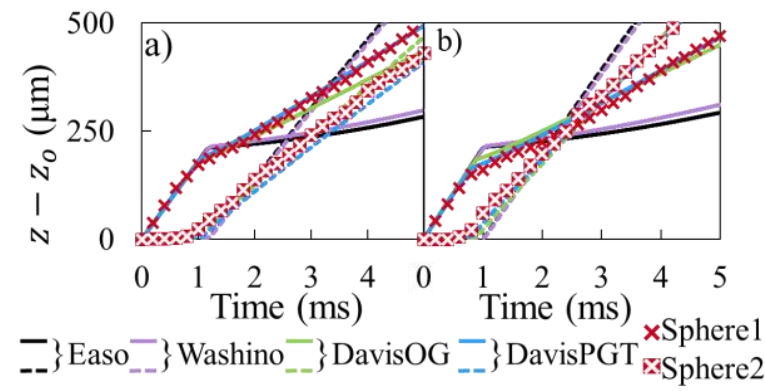

Fig. 3. Change in position during a collision for $2 \mathrm{SS}$ spheres with $3000 \mathrm{cP}$ oil coating with Stokes' numbers of a) 2.7 and b) 3.1, corresponding to an agglomeration collision and a separation collision, respectively. The symbols represent the experimental data. The solid lines represent the striker sphere (1) and the dashed lines represent the initially still sphere (2).

The outcomes of the collision were compared using the effective coefficient of restitution defined as:

$$
e=\frac{v_{1, f}-v_{2, f}}{v_{1, i}}
$$




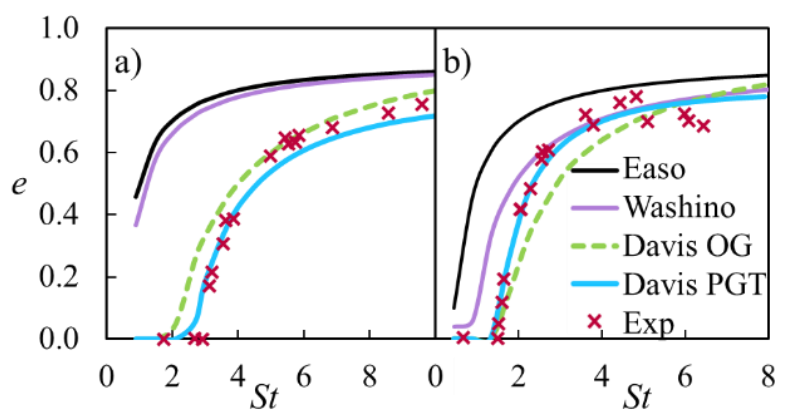

Fig. 4. Measured $e$ as a function of Stokes' number for DEM models and experimental data for a) $3000 \mathrm{cP}$ viscosity oil and SS spheres b) $10000 \mathrm{cP}$ viscosity oil and SS spheres

where $v_{1, f}$ and $v_{2, f}$ are the post collisional velocities of the first and second particles respectively and $v_{1, i}$ is the initial velocity of the striker particle. Figure 4 shows how $e$ changes with increasing Stokes' number for the $\mathrm{SS}$ spheres. In both cases, for $\mathrm{St}<2$ the effective coefficient of restitution is zero and the particles stick together. At about $\mathrm{St}=2$, the two particles separate and the effective coefficient of restitution increases. Similar results were obtained for the CS spheres (not shown), except that the CS results show a slightly greater $e$ owing to their higher dry coefficient of restitution.

The simulation results are also shown in Figure 4. Here the Easo and Washino simulations used the minimum and maximum separation distances that are recommended by LIGGGHTS ${ }^{\mathrm{TM}}$. The Washino model gave approximately similar results to the experimental measurements for the $10000 \mathrm{cP}$ oil, but both models indicated that separation occurred at $\mathrm{St} \approx 1$ for the $3000 \mathrm{cP}$ oil, much lower than was seen experimentally. It was possible to obtain a closer match with the experimental data by fitting $h_{\text {min }}$. However, different $h_{\text {min }}$ values were required as the viscosity changed. The Davis OG model matched fairly well for the lower viscosity case, however the simulation results with the $10000 \mathrm{cP}$ oil were not consistent with the experiments. The Davis PGT model, using a fitted glass transition pressure of $5 \mathrm{MPa}$, produced the best agreement between the experimental data and the simulations. Critically, the Davis PGT model was able to correctly predict the transition from agglomeration to rebound at about $S t=2.5$ and $S t=1.8$, for the $3000 \mathrm{cP}$ and 10000 cP oils, respectively.

\section{Conclusion}

In this work we compared the predictions of four wet granular flow models when simulating collisions between two spheres coated in a thin layer of liquid. The simulation results were compared with high speed camera measurements of similar collisions. Despite the simplicity of the experiment, the collisional outcome varied significantly for each model. The modified glass transition pressure model (Davis PGT) provided the best fit to the experimental results for both viscosities and materials tested. Future experiments are currently underway to investigate the more general applicability of the glass transition pressure model when more particles are studied, or for oblique collisions.

\section{References}

[1] N. Mitarai, F. Nori, Adv. Phys. 55, 1 (2006)

[2] P. Tegzes, T. Vicsek, P. Schiffer, Phys. Rev. Lett. 89, 094301 (2002)

[3] W.R. Ketterhagen, M.T. am Ende, B.C. Hancock, J. Pharm. Sci. 98, 442 (2009)

[4] M. Danczyk, T. Meaclem, M. Mehdizad, D. Clarke, P. Galvosas, L. Fullard, D. Holland, Powder Technol. 372, 671 (2020)

[5] B. Andreotti, Y. Forterre, O. Pouliquen, Granular media: Between Fluid and Solid, Cambridge University Press, 2013

[6] G. Barnocky, R.H. Davis, J. Fluid Mech. 209, 501 (1989)

[7] S.T. Nase, W.L. Vargas, A.A. Abatan, J.J. McCarthy, Powder Technol. 116, 214 (2001)

[8] Liza A. Easo, Liquid Dispersion in Sheared Particulate Material, Purdue University, 2017

[9] H.J. Butt, M. Kappl, Adv. Colloid Interface Sci. 146, 48 (2009)

[10] C.M. Donahue, C.M. Hrenya, R.H. Davis, Soft Condens. Matter. 105, 034501 (2010)

[11] C.M. Donahue, C.M. Hrenya, R.H. Davis, K.J. Nakagawa, A.P. Zelinskaya, G.G. Joseph, J. Fluid Mech. 650, 479 (2010)

[12] B. Buck, S. Heinrich, Adv. Powder Technol. 30 3241 (2019)

[13] P. Darabi, K. Pougatch, M. Salcudean, D. Grecov, Chem. Eng. Sci. 64, 1868 (2009)

[14] K. Washino, E.L. Chan, K. Miyazaki, T. Tsuji, T. Tanaka, Powder Technol. 302, 100 (2016)

[15] A. Goldman, R. Cox, H. Brenner, Chem. Eng. Sci. 22, 637 (1967)

[16] R.H. Davis, J. Serayssol, E.J. Hinch, Fluid Mech. 163, 479 (1986)

[17] G. Barnocky, R.H. Davis, Phys. Fluids. 31, 1324 (1988)

[18] D. Shi, J.J. McCarthy, Powder Technol. 184, 64 (2008)

[19] A.W. Alexander, B. Chaudhuri, A. Faqih, F.J. Muzzio, C. Davies, M.S. Tomassone, Powder Technol. 164, 13 (2006)

[20] A. Anand, J.S. Curtis, C.R. Wassgren, B.C. Hancock, W.R. Ketterhagen, Chem. Eng. Sci. 64, 5268 (2009)

[21] C. Kloss, C. Goniva, A. Hager, S. Amberger, S. Pirker, Prog. Comput. Fluid Dyn. An Int. J. 12, 140 (2012)

[22] R.H. Davis, J. Fluid Mech. 881, 983 (2019)

[23] S. Bair, Friction. 7, 86 (2019) 